\title{
On certain Markov processes attached to exponential functionals of Brownian motion; application to Asian options
}

Catherine Donati-Martin, Raouf Ghomrasni and Marc Yor

\begin{abstract}
We obtain a closed formula for the Laplace transform of the first moment of certain exponential functionals of Brownian motion with drift, which gives the price of Asian options. The proof relies on an identity in law between the average on $[0, t]$ of a geometric Brownian motion and the value at time $t$ of a Markov process, for which we can compute explicitly the resolvent.
\end{abstract}

\section{Introduction.}

The aim of this paper is two-fold:

i) We take up the computation of the value of a continuously averaged Asian option in a Black-Scholes setting, with initial price normalized to 1 , at maturity date $t$, and strike $k$, i.e. $E\left[\left(A_{t}^{a, b}-k\right)^{+}\right]$where $A_{t}^{a, b}=\int_{0}^{t} d s \exp \left(a B_{s}+b s\right)$ and $\left(B_{s} ; s \geq 0\right)$ denotes a one dimensional Brownian motion. The computation of the general price, which involves $r$ the instantaneous risk-free rate easily reduces to the previous computation (for details, see [4, p. 354-355]).

However, our approach in the present paper is very different from 
that in Geman-Yor [3], [4], or Yor [19], which relies very heavily upon Lamperti's representation

$$
\exp \left(B_{t}+\nu t\right)=R^{(\nu)}\left(A_{t}^{(\nu)}\right), \quad t \geq 0,
$$

where $A_{t}^{(\nu)}=\int_{0}^{t} d s \exp \left(2\left(B_{s}+\nu s\right)\right)$, and $\left(R^{(\nu)}(u) ; u \geq 0\right)$ denotes a Bessel process with index $\nu$.

In the present paper, rather than relying on (1.1), we shall use the following remark

$$
A_{t}^{a, b} \stackrel{\text { (law) }}{=} \exp \left(a B_{t}+b t\right) \int_{0}^{t} d s \exp \left(-\left(a B_{s}+b s\right)\right),
$$

for any fixed $t \geq 0$, which is a very particular case of a general identity in law involving the generalized Ornstein-Uhlenbeck processes discussed in Section 2 below.

An important fact is that the right-hand side of (1.2) defines the value at time $t$ of a Markov process $\left(Y_{t}^{a, b} ; t \geq 0\right)$. This remark being made, we write

$$
E\left[\left(A_{t}^{a, b}-k\right)^{+}\right]=E\left[\left(Y_{t}^{a, b}-k\right)^{+}\right],
$$

and we develop the right-hand side of (1.3) using Itô-Tanaka formula.

It turns out than we can compute explicitly the density of the resolvent of $Y^{a, b}$, so that, finally, we obtain another derivation of the main results of Geman-Yor (see [3], [19]).

ii) The second aim of this paper is to present, throughout the text, a more complete view of the bibliography about exponential functionals of Lévy processes than in the Monograph [21]; the incompleteness of the bibliography in [21] is the sole responsability of the third author of the present paper. In particular, we refer to Urbanik [16], [17] for the study of the law of $\int_{0}^{\infty} \exp \left(-u X_{t}\right) d t$ for a positive Lévy process $X$ and to Paulsen [14] and co-authors ([13], [5]) for computations of the laws of randomly discounted integrals $\int_{0}^{\infty} \exp \left(-X_{t}\right) d P_{t}$, where $X$ and $P$ are two independent Lévy processes.

Concerning the price of Asian options, we also mention the work of Rogers-Shi [15] which gives interesting lower and upper bounds for the price.

In a different direction, Leblanc's work [12] deals with the joint law of

$$
\left(\exp \left(B_{t}+\nu t\right), \int_{0}^{t} \exp \left(B_{S}+\nu s\right) d s, \int_{0}^{t} \exp 2\left(B_{s}+\nu s\right) d s\right) .
$$


We now hope that, together with the references found in [21] concerning exponential functionals of Lévy processes, this paper offers a more reasonably complete bibliography (than in [21]). Needless to say, any new omission of relevant work is not intended.

\section{On generalized Ornstein-Uhlenbeck processes.}

It is a remarkable fact that, if $\left\{\left(\xi_{t}, \eta_{t}\right) ; t \geq 0\right\}$ is a two-dimensional Lévy process with respect to a filtration $\left(\mathcal{F}_{t}\right)$, then the process defined by

$$
X_{t}=\exp \left(\xi_{t}\right)\left(x+\int_{0}^{t} \exp \left(-\xi_{s^{-}}\right) d \eta_{s}\right)
$$

is also a Markov process. Cases of particular interest involve independent $\xi$ and $\eta$, but this independence hypothesis is not necessary.

Some of these processes have been studied in the literature. The case where $\xi_{s}=\lambda s$ and $\eta$ is a Brownian motion gives the usual Ornstein-Uhlenbeck process of parameter $\lambda$. Hadjiev [7] considers the case where $\xi_{s}=\lambda s$ and $\eta$ is a Lévy process without positive jumps and determines the distribution of the hitting times for $X$. Gravereaux [6] studies the case where $\eta$ is a $d$-dimensional Lévy process and $\xi_{s}=s h$ where $h$ is a linear map on $\mathbb{R}^{d}$, and looks for the existence of an invariant measure. We also refer to Jurek [9] for the condition on $\eta$ insuring the existence of $\int_{0}^{\infty} \exp (\lambda s) d \eta_{s}$ for $\lambda<0$ and to Jacod [8] for the study of (2.1) when the initial condition $x$ is replaced by an anticipating random variable.

Yor [22] considers the process $X$ for $\xi$ and $\eta$ two independent Brownian motions with respective drifts $\mu$ and $\nu$ and deduces from Proposition 2.1 below the law of a subordinated perpetuity, a result already obtained by Paulsen [14] by a different method.

We also mention the work of de Haan-Karandikar [10] where the Markov process $X$ appears as the solution of a "SDE" of the form

$$
X_{t}=A_{t}^{s} X_{s}+B_{t}^{s}, \quad s \leq t,
$$

for random variables $\left\{A_{t}^{s}, B_{t}^{s} ; s \leq t\right\}$ satisfying compatibility conditions and certain independence and stationarity properties.

These Markov processes are related to exponential functionals of Lévy processes via the following: 
Proposition 2.1. Let $\xi$ and $\eta$ be two independent Lévy processes, then for fixed $t$,

$$
\begin{aligned}
\left(\exp \left(\xi_{t}\right), \exp \left(\xi_{t}\right)\right. & \left.\int_{0}^{t} \exp \left(-\xi_{s^{-}}\right) d \eta_{s}\right) \\
& \stackrel{\text { (law) }}{=}\left(\exp \left(\xi_{t}\right), \int_{0}^{t} \exp \left(\xi_{s^{-}}\right) d \eta_{s}\right) .
\end{aligned}
$$

This identity follows from the invariance by time reversal of the distribution of a Lévy process. We refer to Carmona-Petit-Yor in [21] for applications of this result.

Corollary 2.1. Consider the Lévy process

$$
\xi_{t}=-\left(c t+\sigma B_{t}+\tau_{t}^{+}-\tau_{t}^{-}\right)
$$

where $\left(\tau_{t}^{ \pm} ; t \geq 0\right)$ are subordinators without drift and Lévy measures $\nu_{ \pm} ; B$ is a Brownian motion and the processes $B, \tau^{+}, \tau^{-}$are independent. We denote by $\Phi(\lambda)$ the Lévy exponent of $\xi$ determined by $E\left(\exp \left(\lambda \xi_{t}\right)\right)=\exp (-t \Phi(\lambda))$.

Let

$$
A_{t}=\int_{0}^{t} \exp \left(\xi_{s}\right) d s
$$

and

$$
X_{t}=\exp \left(\xi_{t}\right) \int_{0}^{t} \exp \left(-\xi_{s}\right) d s .
$$

$T_{\alpha}$ denotes an exponential variable of parameter $\alpha$, independent of $\xi$.

i) The law $\mu_{\alpha}$ of $A_{T_{\alpha}}$ satisfies

$$
\mu_{\alpha}=\frac{1}{\alpha} L^{*} \mu_{\alpha}, \quad \text { on }(0, \infty)
$$

where $L$ denotes the infinitesimal generator of the Markov process $X$.

ii) In particular, the moments of $A_{T_{\alpha}}$ satisfy

$$
E\left[A_{T_{\alpha}}^{m}\right]=\frac{m}{\alpha+\Phi(m)} E\left[A_{T_{\alpha}}^{m-1}\right]
$$

for $m>0$ and $\alpha+\Phi(m)>0$. 
iii) Finally, if $A_{\infty}<\infty$ almost surely, then $\mu_{0}$, the law of $A_{\infty}$, solves $L^{*} \mu_{0}=0$.

Proof. i) From Proposition 2.1, for $f \in \operatorname{Dom}(L)$,

$$
\begin{aligned}
E\left[f\left(A_{t}\right)\right] & =E\left[f\left(X_{t}\right)\right] \\
& =f(0)+E\left[\int_{0}^{t} L f\left(X_{s}\right) d s\right] \\
& =f(0)+\int_{0}^{t} E\left[L f\left(A_{s}\right)\right] d s .
\end{aligned}
$$

Thus,

$$
\begin{aligned}
E\left[f\left(A_{T_{\alpha}}\right)\right] & =f(0)+\alpha \int_{0}^{\infty} d t \exp (-\alpha t) \int_{0}^{t} E\left[L f\left(A_{s}\right)\right] d s \\
& =f(0)+\int_{0}^{\infty} d t \exp (-\alpha t) E\left[L f\left(A_{t}\right)\right]=\frac{1}{\alpha} E\left[L f\left(A_{T_{\alpha}}\right)\right]
\end{aligned}
$$

proving $(2.3)$, as we restrict $f$ to $C_{K}^{\infty}((0, \infty))$, and use integration by parts.

ii) (2.4) has already been obtained by Carmona-Petit-Yor in [21]. We give another proof relying on (2.3).

The generator $L$ of $X$ is given by (see [21, p. 81])

$$
\begin{aligned}
L f(x)= & \frac{\sigma^{2}}{2} x^{2} f^{\prime \prime}(x)+\left(\left(\frac{\sigma^{2}}{2}-c\right) x+1\right) f^{\prime}(x) \\
& -\int_{0}^{x} f^{\prime}(u) \bar{\nu}_{+}\left(\ln \left(\frac{x}{u}\right)\right) d u+\int_{x}^{\infty} f^{\prime}(u) \bar{\nu}_{-}\left(\ln \left(\frac{u}{x}\right)\right) d u
\end{aligned}
$$

where $\bar{\nu}$ is the tail of $\nu$. (We point out a misprint in the formula given in $[21$, p. 81$]$ where the sign minus before the coefficient of $f^{\prime \prime}$ must be deleted).

An easy computation shows that if $f_{m}(x):=x^{m}, m>0$, then

$$
\begin{aligned}
L f_{m}(x)= & \left(\frac{\sigma^{2}}{2} m^{2}-c m+\int_{0}^{\infty}(\exp (-m z)-1) \bar{\nu}_{+}(d z)\right. \\
& \left.+\int_{0}^{\infty}(\exp (m z)-1) \bar{\nu}_{-}(d z)\right) f_{m}(x)+m f_{m-1}(x) \\
= & -\Phi(m) f_{m}(x)+m f_{m-1}(x) .
\end{aligned}
$$


Thus, from (2.3),

$$
E\left[A_{T_{\alpha}}^{m}\right]=\frac{1}{\alpha} E\left[m A_{T_{\alpha}}^{m-1}-\Phi(m) A_{T_{\alpha}}^{m}\right]
$$

hence

$$
E\left[A_{T_{\alpha}}^{m}\right]=\frac{m}{\alpha+\Phi(m)} E\left[A_{T_{\alpha}}^{m-1}\right]
$$

iii) It suffices to multiply both sides of (2.3) by $\alpha$ and to let $\alpha$ converge to 0 .

3. Application to the computation of the price of Asian options.

We take up (1.3) again, i.e.

$$
E\left[\left(A_{t}^{a, b}-k\right)^{+}\right]=E\left[\left(Y_{t}^{a, b}-k\right)^{+}\right],
$$

where

$$
Y_{t}^{a, b}(x)=\exp \left(a B_{t}+b t\right)\left(x+\int_{0}^{t} d s \exp \left(-\left(a B_{s}+b s\right)\right)\right)
$$

is a Markov process and we write simply $Y_{t}^{a, b}$ for $Y_{t}^{a, b}(0)$. It may be worth mentioning that these processes come up as an important example throughout Arnold's book [1].

Proposition 3.1. The process $Y_{t}^{a, b}(x)$ is the solution of the equation

$$
Y_{t}=x+a \int_{0}^{t} d B_{u} Y_{u}+\int_{0}^{t} d u\left(\left(\frac{a^{2}}{2}+b\right) Y_{u}+1\right)
$$


Proof. This is immediate, using Itô's formula, and Fubini's theorem.

By scaling, we may and we shall assume that $a^{2} / 2=1$ and we set $Y_{\nu}=Y^{\sqrt{2}, \nu}$.

Theorem 3.1. We denote by $U_{\alpha}$ the resolvent of the Markov process $Y_{\nu}$, that is

$$
U_{\alpha} f(x)=\int_{0}^{\infty} \exp (-\alpha t) E_{x}\left[f\left(Y_{\nu}(t)\right)\right] d t
$$

Then, the resolvent $U_{\alpha}$ admits a density which is given by

$$
\begin{aligned}
& u_{\alpha}(x, y)=\frac{\Gamma\left(\frac{\nu+\mu}{2}\right)}{\Gamma(1+\mu)}\left(\mathbf{1}_{[0, x[}(y)\left(\frac{1}{y}\right)^{1-\nu} \exp \left(-\frac{1}{y}\right) \varphi_{1}(x) \varphi_{2}(y)\right. \\
& \left.+\mathbf{1}_{[x, \infty[}(y)\left(\frac{1}{y}\right)^{1-\nu} \exp \left(-\frac{1}{y}\right) \varphi_{2}(x) \varphi_{1}(y)\right),
\end{aligned}
$$

for $x, y>0$ where

$$
\begin{aligned}
& \varphi_{1}(x)=\left(\frac{1}{x}\right)^{(\nu+\mu) / 2} \Phi\left(\frac{\nu+\mu}{2} ; 1+\mu ; \frac{1}{x}\right), \\
& \varphi_{2}(x)=\left(\frac{1}{x}\right)^{(\nu+\mu) / 2} \Psi\left(\frac{\nu+\mu}{2} ; 1+\mu ; \frac{1}{x}\right),
\end{aligned}
$$

with $\mu=\sqrt{\nu^{2}+4 \alpha}$ and $\Phi$ and $\Psi$ denote the confluent hypergeometric functions of first and second kind (see Lebedev [11, Section 9.9]).

Proof. Let $f$ be a bounded function on $\mathbb{R}_{+}$. The function $u(x):=$ $U_{\alpha} f(x)$ solves the differential equation $(\alpha I-L) u(x)=f(x)$ where $L$ denotes the infinitesimal generator of $Y_{\nu}$. Thus, $u$ is the bounded solution of

$$
x^{2} \frac{d^{2}}{d x^{2}} u(x)+((1+\nu) x+1) \frac{d}{d x} u(x)-\alpha u(x)+f(x)=0 .
$$

Let us consider the homogeneous equation associated to (3.4)

$$
x^{2} y^{\prime \prime}+((1+\nu) x+1) y^{\prime}-\alpha y=0 \text {. }
$$


Then, $\varphi_{1}$ and $\varphi_{2}$, given by (3.2) and (3.3) are two independent solutions of (3.5). Moreover, $\varphi_{1}$ is bounded near $+\infty$ and $\varphi_{2}$ is bounded near $0\left(\varphi_{2}(0)=1\right)$. Now, we are looking for a solution of (3.4) of the form $u(x)=\alpha_{1}(x) \varphi_{1}(x)+\alpha_{2}(x) \varphi_{2}(x)$ with

$$
\left\{\begin{array}{l}
\alpha_{1}^{\prime}(x) \varphi_{1}(x)+\alpha_{2}^{\prime}(x) \varphi_{2}(x)=0 \\
\alpha_{1}^{\prime}(x) \varphi_{1}^{\prime}(x)+\alpha_{2}^{\prime}(x) \varphi_{2}^{\prime}(x)=-\frac{f(x)}{x^{2}}
\end{array}\right.
$$

Then,

$$
\alpha_{1}^{\prime}(x)=\frac{f(x) \varphi_{2}(x)}{W\left(\varphi_{1}, \varphi_{2}\right)(x) x^{2}}, \quad \alpha_{2}^{\prime}(x)=\frac{-f(x) \varphi_{1}(x)}{W\left(\varphi_{1}, \varphi_{2}\right)(x) x^{2}},
$$

where the Wronskian $W\left(\varphi_{1}, \varphi_{2}\right)(x)$ is given by

$$
W\left(\varphi_{1}, \varphi_{2}\right)(x)=\frac{\Gamma(1+\mu)}{\Gamma\left(\frac{\nu+\mu}{2}\right)}\left(\frac{1}{x}\right)^{1+\nu} \exp \left(\frac{1}{x}\right)
$$

(see $[11,(9.10 .10)])$. Using the boundary conditions on $\varphi_{1}, \varphi_{2}$, the bounded solution of (3.4) is given by

$$
\begin{aligned}
u(x)= & \left(\int_{0}^{x} \frac{f(t) \varphi_{2}(t)}{W\left(\varphi_{1}, \varphi_{2}\right)(t) t^{2}} d t\right) \varphi_{1}(x) \\
& +\left(\int_{x}^{\infty} \frac{f(t) \varphi_{1}(t)}{W\left(\varphi_{1}, \varphi_{2}\right)(t) t^{2}} d t\right) \varphi_{2}(x)
\end{aligned}
$$

This gives formula (3.1).

Corollary 3.1. Let $T_{\alpha}$ be an exponential time with parameter $\alpha$ independent from $B$; then the density of $A_{T_{\alpha}}^{\sqrt{2}, \nu}$ is given by

$$
\begin{aligned}
k_{\nu}(x)= & \alpha \frac{\Gamma\left(\frac{\nu+\mu}{2}\right)}{\Gamma(1+\mu)}\left(\frac{1}{x}\right)^{1+(\mu-\nu) / 2} \\
& \cdot \exp \left(-\frac{1}{x}\right) \Phi\left(\frac{\nu+\mu}{2} ; 1+\mu ; \frac{1}{x}\right) .
\end{aligned}
$$

For $\alpha>\nu+1$, we have

$$
\begin{aligned}
E\left[\left(A_{T_{\alpha}}^{\sqrt{2}, \nu}-k\right)^{+}\right]= & \frac{\Gamma\left(\frac{\nu+\mu}{2}+1\right) \Gamma\left(\frac{\mu-\nu}{2}-1\right)}{\Gamma(1+\mu) \Gamma\left(\frac{\mu-\nu}{2}\right)} \\
& \cdot k^{1-(\mu-\nu) / 2} \exp \left(-\frac{1}{k}\right) \Phi\left(\frac{\nu+\mu}{2}+2 ; 1+\mu ; \frac{1}{k}\right) .
\end{aligned}
$$


Proof. 1) From (1.3), the distribution of $A_{T_{\alpha}}^{\sqrt{2}, \nu}$ is the same as the distribution of $Y_{\nu}\left(T_{\alpha}\right)$, that is $\alpha u_{\alpha}(0, y) d y$. Thus, (3.7) follows from (3.1) and $\varphi_{2}(0)=1$.

2) $E\left[\left(A_{T_{\alpha}}^{\sqrt{2}, \nu}-k\right)^{+}\right]=\alpha U_{\alpha} f_{k}(0)$ for $f_{k}(x)=(x-k)^{+}$. From $(3.1)$,

$$
\begin{aligned}
U_{\alpha} f_{k}(0)=\frac{\Gamma\left(\frac{\nu+\mu}{2}\right)}{\Gamma(1+\mu)} \int_{k}^{\infty}(t-k)\left(\frac{1}{t}\right)^{1+(\mu-\nu) / 2} \\
\cdot \exp \left(-\frac{1}{t}\right) \Phi\left(\frac{\nu+\mu}{2} ; 1+\mu ; \frac{1}{t}\right) d t
\end{aligned}
$$

and the integral converges for $\alpha>\nu+1$. Now, we have the following relation

$$
\begin{aligned}
& \int_{k}^{\infty}(t-k)\left(\frac{1}{t}\right)^{1+(\mu-\nu) / 2} \exp \left(-\frac{1}{t}\right) \Phi\left(\frac{\nu+\mu}{2} ; 1+\mu ; \frac{1}{t}\right) d t \\
& (3.9)=\frac{\Gamma\left(\frac{\mu-\nu}{2}-1\right)}{\Gamma\left(\frac{\mu-\nu}{2}+1\right)} k^{1-(\mu-\nu) / 2} \exp \left(-\frac{1}{k}\right) \Phi\left(\frac{\nu+\mu}{2}+2 ; 1+\mu ; \frac{1}{k}\right)
\end{aligned}
$$

(see Lebedev [11, 21. p. 279] and Yor [19, Chapter 6] for a probabilistic proof of this relation) and (3.8) follows.

REMARK. 1) Formula (3.8) yields the result of Geman and Yor [3, (2)] since, by scaling,

$$
E\left[\left(A_{T_{\lambda}}^{(\nu)}-\frac{1}{2 x}\right)^{+}\right]=\frac{1}{2} E\left[\left(A_{T_{\lambda / 2}}^{\sqrt{2}, \nu}-\frac{1}{x}\right)^{+}\right] .
$$

2) We can decompose the right-hand side of (1.3) using Itô-Tanaka formula

$$
E\left[\left(Y_{t}-k\right)^{+}\right]=\int_{0}^{t} d s E\left[\left(\left(\frac{a^{2}}{2}+b\right) Y_{s}+1\right) \mathbf{1}_{\left\{Y_{s}>k\right\}}\right]+\frac{1}{2} E\left[L_{t}^{k}\right],
$$

where $\left(L_{t}^{k} ; t \geq 0\right)$ denotes the local time of $Y^{a, b}$ at level $k$. Thus, the quantity $C^{a, b}(t, k):=E\left[\left(Y_{t}-k\right)^{+}\right]$can be decomposed as

$$
C^{a, b}(t, k)=\left(\frac{a^{2}}{2}+b\right) C_{1}^{a, b}(t, k)+C_{2}^{a, b}(t, k)+\frac{1}{2} C_{3}^{a, b}(t, k),
$$


where

$$
\left\{\begin{aligned}
C_{1}^{a, b}(t, k) & =\int_{0}^{t} d s E\left[Y_{s} \mathbf{1}_{\left\{Y_{s}>k\right\}}\right] \\
C_{2}^{a, b}(t, k) & =\int_{0}^{t} d s E\left[\mathbf{1}_{\left\{Y_{s}>k\right\}}\right] \\
C_{3}^{a, b}(t, k) & =E\left[L_{t}^{k}\right]
\end{aligned}\right.
$$

We restrict ourselves to the case $a=\sqrt{2}, b=\nu$ and we delete the superscripts in $C$.

From Proposition 3.1, we can compute the Laplace transform

$$
\int_{0}^{\infty} d t \exp (-\alpha t) C_{i}(t, k)
$$

for $i=1,2,3$. For $i=3$, from the occupation density formula

$$
\int_{0}^{t} f\left(Y_{s}\right) d\langle Y\rangle_{s}=\int_{0}^{\infty} f(y) L_{t}^{y} d y,
$$

it follows that

$$
\begin{aligned}
E\left[L_{T_{\alpha}}^{y}\right] & =2 y^{2} u_{\alpha}(0, y) \\
& =2 \frac{\Gamma\left(\frac{\nu+\mu}{2}\right)}{\Gamma(1+\mu)} y^{1-(\mu-\nu) / 2} \exp \left(-\frac{1}{y}\right) \Phi\left(\frac{\nu+\mu}{2} ; 1+\mu ; \frac{1}{y}\right) .
\end{aligned}
$$

Now,

$$
\begin{aligned}
E[ & \left.C_{2}\left(T_{\alpha}, k\right)\right] \\
& =\int_{0}^{\infty} \exp (-\alpha t) E\left[\mathbf{1}_{\left\{Y_{t}>k\right\}}\right] d t \\
& =U_{\alpha} \mathbf{1}_{\{x>k\}}(0) \\
& =\frac{\Gamma\left(\frac{\nu+\mu}{2}\right)}{\Gamma(1+\mu)} \int_{k}^{\infty}\left(\frac{1}{t}\right)^{1+(\mu-\nu) / 2} \exp \left(-\frac{1}{t}\right) \Phi\left(\frac{\nu+\mu}{2} ; 1+\mu ; \frac{1}{t}\right) d t .
\end{aligned}
$$

Now, the following relation holds (see Lebedev [11, 21. p. 279])

$$
\begin{aligned}
& \int_{k}^{\infty}\left(\frac{1}{t}\right)^{1+(\mu-\nu) / 2} \exp \left(-\frac{1}{t}\right) \Phi\left(\frac{\nu+\mu}{2} ; 1+\mu ; \frac{1}{t}\right) d t \\
& \quad=\frac{2}{\mu-\nu} k^{(\nu-\mu) / 2} \exp \left(-\frac{1}{k}\right) \Phi\left(\frac{\nu+\mu}{2}+1 ; 1+\mu ; \frac{1}{k}\right) .
\end{aligned}
$$


In the same way,

$$
\begin{aligned}
& E\left[C_{1}\left(T_{\alpha}, k\right)\right] \\
& =\frac{\Gamma\left(\frac{\nu+\mu}{2}\right)}{\Gamma(1+\mu)} \int_{k}^{\infty} t\left(\frac{1}{t}\right)^{1+(\mu-\nu) / 2} \exp \left(-\frac{1}{t}\right) \Phi\left(\frac{\nu+\mu}{2} ; 1+\mu ; \frac{1}{t}\right) d t,
\end{aligned}
$$

which can be expressed in term of $\Phi((\nu+\mu) / 2+1 ; 1+\mu ; 1 / k)$ and $\Phi((\nu+\mu) / 2+2 ; 1+\mu ; 1 / k)$ using the two relations (3.9) and (3.10).

In terms of confluent hypergeometric functions, the equality

$$
E\left[C\left(T_{\alpha}, k\right)\right]=(1+\nu) E\left[C_{1}\left(T_{\alpha}, k\right)\right]+E\left[C_{2}\left(T_{\alpha}, k\right)\right]+\frac{1}{2} E\left[C_{3}\left(T_{\alpha}, k\right)\right]
$$

corresponds to the recurrence relation

$$
(b-a) \Phi(a-1 ; b ; z)+(2 a-b+z) \Phi(a ; b ; z)-a \Phi(a+1 ; b ; z)=0
$$

$(\operatorname{see}[11,(9.9 .10)])$.

\section{Some finite dimensional Markov processes.}

It was shown in [2], [20] that, for $a \neq 0$, and $b>0$,

(4.1) the variable $\int_{0}^{\infty} d s \exp \left(a B_{s}-b s\right)$ is distributed as $\frac{2}{a^{2} Z_{2 b / a^{2}}}$,

where $Z_{\nu}$ is a gamma variable with parameter $\nu$, i.e.

$$
P\left(Z_{\nu} \in d t\right)=\frac{d t t^{\nu-1} e^{-t}}{\Gamma(\nu)} .
$$

However, in [21], the joint law of

$$
\left\{\int_{0}^{\infty} d s \exp \left(a_{i} B_{s}-b_{i} s\right), i=1,2, \cdots, n\right\}
$$

for different constants $a_{i}, b_{i}$ could not be obtained.

In this section, using (1.2), we can express this law as the invariant measure of a Markov process. Indeed, we consider jointly the onedimensional Markov processes $\left(Y_{t}^{(i)} ; t \geq 0\right)$ defined as

$$
Y_{t}^{(i)}=\int_{0}^{t} d s \exp \left(\xi_{t}^{(i)}-\xi_{s}^{(i)}\right), \quad t \geq 0
$$


where $\xi_{t}^{(i)}=a_{i} B_{t}+b_{i} t$, and $\left(B_{t}\right)$ is a one-dimensional Brownian motion.

Of course, these processes are not independent, and jointly, they constitute a $\left(\mathbb{R}_{+}\right)^{n}$-valued Markov process, specified in the following:

Thorem 4.1. The process $Y_{t} \equiv\left(Y_{t}^{(1)}, \ldots, Y_{t}^{(n)}\right), t \geq 0$ is a Markov process, whose infinitesimal generator coincides on $C^{2}\left((0, \infty)^{n}\right)$ with

$$
\begin{aligned}
L= & \frac{1}{2}\left(\sum_{i=1}^{n} a_{i}^{2} y_{i}^{2} \frac{\partial^{2}}{\partial y_{i}^{2}}+2 \sum_{i<j} a_{i} a_{j} y_{i} y_{j} \frac{\partial^{2}}{\partial y_{i} \partial y_{j}}\right) \\
& +\sum_{i=1}^{n}\left(\left(\frac{a_{i}^{2}}{2}+b_{i}\right) y_{i}+1\right) \frac{\partial}{\partial y_{i}} .
\end{aligned}
$$

We are now interested in the case where $b_{i}<0$, for every $i$. In this case, since

$$
Y_{t}^{(i)} \stackrel{(\text { law })}{=} \int_{0}^{t} d s \exp \left(\xi_{s}^{(i)}\right)
$$

the vector $Y_{t} \equiv\left(Y_{t}^{(1)}, \cdots, Y_{t}^{(n)}\right)$ converges in law, towards

$$
U=\left(U^{(1)}, U^{(2)}, \ldots, U^{(n)}\right),
$$

where $U^{(i)}=\int_{0}^{\infty} d s \exp \left(\xi_{s}^{(i)}\right)$

Our aim now is to describe $\mu$, the joint law of the random vector $U$.

Just as in Carmona-Petit-Yor in [21], we note that $\mu$ is the unique invariant measure of the Markov process $Y$; hence, it satisfies: $\mu L=0$, i.e.

$$
\text { for all } f \in C_{b}^{2}\left(\left(\mathbb{R}_{+}\right)^{n}\right), \quad\langle\mu, L f\rangle=0 \text {. }
$$

Let us assume that $\mu(d y)=k(y) d y$, where $y$ denotes the generic element in $\left(\mathbb{R}_{+}\right)^{n}$, and $d y$ is Lebesgue's measure on $\left(\mathbb{R}_{+}\right)^{n}$.

Now, from (4.2), it follows that

$$
\frac{1}{2}\left(\sum_{i=1}^{n} a_{i}^{2} \frac{\partial^{2}}{\partial y_{i}^{2}}\left(y_{i}^{2} k(y)\right)+2 \sum_{i<j} a_{i} a_{j} \frac{\partial^{2}}{\partial y_{i} \partial y_{j}}\left(y_{i} y_{j} k(y)\right)\right)
$$

$$
-\sum_{i=1}^{n}\left(\left(\frac{a_{i}^{2}}{2}+b_{i}\right) \frac{\partial}{\partial y_{i}}\left(y_{i} k(y)\right)+\frac{\partial}{\partial y_{i}}(k(y))\right)=0 .
$$


We check that in the case $n=1$, we recover the result (4.1). Indeed, the density $(k(u), u>0)$ of $X \equiv c / Z_{\nu}$ is

$$
k(u)=\frac{c^{\nu}}{\Gamma(\nu) u^{\nu+1}} \exp \left(-\frac{c}{u}\right)
$$

where we have denoted $c=2 / a^{2}$, and $\nu=2 b / a^{2}$.

On the other hand, for $n=1$, and $a_{1}=a, b_{1}=-b$, (4.3) becomes

$$
\left(y^{2} k(y)\right)^{\prime \prime}-(1+\nu)(y k(y))^{\prime}-c k^{\prime}(y)=0
$$

and we easily verify that the density $k$ defined above solves (4.4).

REMARK. 1) Unfortunately, except in the case $n=1$, it does not seem easy to solve (4.2), i.e. to find explicitly the density of $U$. It may be easier to find the Laplace transform $\psi$ of $U$. From (4.3), we can easily deduce the equation satisfied by $\psi$, that is

$$
\widehat{L} \psi(x)=\left(\sum_{1}^{n} x_{i}\right) \psi(x), \quad x \in \mathbb{R}_{+}^{n},
$$

where

$\widehat{L}:=\frac{1}{2}\left(\sum_{i=1}^{n} a_{i}^{2} y_{i}^{2} \frac{\partial^{2}}{\partial y_{i}^{2}}+2 \sum_{i<j} a_{i} a_{j} y_{i} y_{j} \frac{\partial^{2}}{\partial y_{i} \partial y_{j}}\right)+\sum_{i=1}^{n}\left(\frac{a_{i}^{2}}{2}+b_{i}\right) y_{i} \frac{\partial}{\partial y_{i}}$

with the boundary conditions $\psi(0)=1$ and $\lim _{x \rightarrow \infty} \psi(x)=0$.

For $n=1,(4.5)$ is a particular case of Theorem 3.3 of Paulsen [14] where it is shown that the Laplace transform of a randomly discounted integral solves an integro-differential equation.

2) For $n=2$, the Laplace transform of $U=\left(U^{(1)}, U^{(2)}\right)$ is obtained in $[20],[22]$ in the two particular cases where

1) $a_{1}=-a_{2}=2$ and $b_{1}=b_{2}=-1$.

2) $a_{1}=2 a_{2}=2$ and $b_{1}=2 b_{2}=\mu(\mu<0)$.

\section{References.}

[1] Arnold, L., Random dynamical systems. Springer, 1998. 
[2] Dufresne, D., The distribution of perpetuity, with applications to risk theory and pension funding. Scand. Act. Journal 1-2 (1990), 39-79.

[3] Geman, H., Yor, M., Quelques relations entre processus de Bessel, options asiatiques et fonctions confluentes hypergéométriques. C. R. Acad. Sci. Paris 314 (1992), 471-474.

[4] Geman, H., Yor, M., Bessel processes, Asian options and perpetuities. Math. Finance 3 (1993), 349-375.

[5] Gjessing, H. K., Paulsen, J., Present value distributions with applications to ruin theory and stochastic equations. Stoch. Proc. and their Appl. 71 (1997), 123-144.

[6] Gravereaux, J. B., Probabilités sur $\mathbb{R}^{d}$ et équations différentielles stochastiques linéaires. Séminaire de Probabilités de Rennes, 1982.

[7] Hadjiev, D. I., The first passage problem for generalized Ornstein-Uhlenbeck processes with non positive jumps. Sém. Probab. XIX, Lecture Notes in Math. 1123 (1985), Springer.

[8] Jacod, J., Grossissement de filtration et processus d'Ornstein-Uhlenbeck généralisé. In: Grossissements de filtrations: Exemples et applications. Lecture Notes in Math. 1118, Springer (1985), 197-304.

[9] Jurek, Z. J., An integral representation of operator-self-decomposable random variables. Bull. Ac. Polonaise des Sc. XXX (1982), 385-393.

[10] de Haan, L., Karandikar, R. L., Embedding a stochastic difference equation into a continuous-time process. Stoc. Proc. and their Appl. 32 (1989), 225-235.

[11] Lebedev, N. N., Special functions and their applications. Dover Publications, 1972.

[12] Leblanc, B., Une approche unifiée pour une forme exacte du prix d'une option dans les différents modèles à volatilité stochastique. Stochastics and Stochastic Reports 57 (1996), 1-35.

[13] Nilsen, T., Paulsen, J., On the distribution of a randomly discounted compound Poisson process. Stoch. Proc. and their Appl. 61 (1996), 305-310.

[14] Paulsen, J., Risk theory in a stochastic economic environment. Stoch. Proc. and their Appl. 46 (1993), 327-341.

[15] Rogers, L. C. G., Shi, Z., The value of an Asian option. Journal of Appl. Prob. 32 (1995), 1077-1088.

[16] Urbanik, K., Functionals on transient stochastic processes with independent increments. Studia Math. 103 (1992), 299-315.

[17] Urbanik, K., Infinite divisibility of some functionals on stochastic processes. Prob. and Math. Stat. 15 (1995), 493-513.

[18] Wong, E., The construction of a class of stationary Markov processes. 
Amer. Math. Soc. Proceedings of the 16th Symposium of Appl. Math. (1964), 264-276.

[19] Yor, M., Some Aspects of Brownian Motion. Part I: Some Special Functionals. Lectures in Mathematics. E.T.H. Zürich, Birkhäuser, 1992.

[20] Yor, M., Sur certaines fonctionnelles exponentielles du mouvement brownien réel. Journal of App. Probab. 29 (1992), 202-208.

[21] Yor, M., ed., Exponential functionals and principal values related to Brownian motion. Biblioteca de la Revista Matematica Iberoamericana, 1997.

[22] Yor, M., Interpretation in terms of Brownian and Bessel meanders of the distribution of a subordinated Brownian perpetuity. To appear in a volume on Lévy processes, edited by Th. Mikosch. Birkaüser, 2001.

Recibido: 21 de mayo de 1.999

Catherine Donati-Martin Laboratoire de Statistique et Probabilités

UMR CNRS 5583

Université P. Sabatier 118, route de Narbonne 31062 Toulouse Cedex, FRANCE donati@cict.fr

and

Raouf Ghomrasni, Marc Yor Laboratoire de Probabilités et Modèles aléatoires UMR CNRS 7599, Tour 56, $3^{e}$ étage Université Paris VI 4, place Jussieu 75252 Paris Cedex 05, FRANCE secret@proba.jussieu.fr 